\title{
Um Problema de Interferência Quântica em Nanoestruturas Metálicas ${ }^{1}$
}

D.G. TEIXEIRA 2 , A.C. DE CASTRO BARBOSA, Instituto de Matemática e Estatística, UERJ - Universidade do Estado do Rio de Janeiro, 20.550-013 Rio de Janeiro, RJ, Brasil.

M.V.T. COSTA, Instituto de Aplicação, UERJ - Universidade do Estado do Rio de Janeiro, 20.261-232 Rio de Janeiro, RJ, Brasil.

\begin{abstract}
Resumo. Um problema relacionado ao fenômeno de interferência quântica em um sistema nanoestruturado é investigado através do estudo da segregação de impurezas substitucionais. Tais sistemas são formados pela justaposição de camadas de átomos de metais de transição. A segregação de impurezas substitucionais é tratada a partir do cálculo da variação da energia eletrônica total do sistema, considerando as posições da impureza relativamente à superfície. Empregamos o modelo de ligações fortes, com apenas um orbital por sítio, sendo os potenciais das impurezas determinados de modo a satisfazer a neutralidade de carga através da regra de soma de Friedel. Consideramos uma rede cúbica simples e as direções (100) e (110).
\end{abstract}

Palavras-chave. Segregação, Multicamadas, Funções de Green.

\section{Introdução}

Desde o início da década de 1980, sistemas metálicos de baixa dimensionalidade, tais como superfícies, filmes e multicamadas tem recebido uma atenção especial por apresentarem características peculiares e extremamente promissoras em termos de aplicações tecnológicas. Além disso, representam um novo desafio teórico e experimental dos conceitos físicos estabelecidos. A pesquisa nestes sistemas é um empreendimento interdisciplinar, envolvendo diversas áreas do conhecimento tais como Matemática, Física, Química e Ciência dos Materiais.

Multicamadas metálicas são materiais formados por camadas de metais dispostas paralelamente umas às outras. Os progressos obtidos na preparação desses sistemas nanoestruturados estão fortemente relacionados ao desenvolvimento das técnicas de alto vácuo [16], das técnicas de crescimento e de análise de suas estruturas. Os métodos de crescimento mais conhecidos são o sputtering (pulverização) [19] e o MBE (epitaxia por feixe molecular) [17]. Na análise da composição desses sistemas, diversas técnicas experimentais podem ser empregadas, dentre as quais

\footnotetext{
${ }^{1}$ Financiado pela FAPERJ. Uberlândia, MG, Brasil [11].

${ }^{2}$ nellet@ig.com.br.

3 accb@ime.uerj.br.

${ }^{4}$ mvtc@uerj.br.
} 
podemos destacar a difração de raios-X, que fornece informações sobre o estado cristalográfico da amostra, espectroscopia Auger e Mössbauer, usadas principalmente na verificação da composição química e do perfil da concentração de determinados elementos.

A presença de defeitos, tais como impurezas ou imperfeições de interface, podem afetar profundamente a estrutura eletrônica destes sistemas, o que por sua vez acarreta em mudanças de suas propriedades físicas $[6,13]$.

Foi observado por Tsong e colaboradores o aparecimento de um comportamento oscilatório na composição de ligas próximo à superfície como, por exemplo, em ligas metálicas do tipo Pt-Rh e Pt-Ni, em que as composições do Rh e do Ni variam plano a plano, a partir da superfície, de forma decrescente em relação à concentração do volume $[20,21]$.

Nesse trabalho, procuramos explicar esse comportamento oscilatório considerando a variação da energia eletrônica total. Esta variação decorre da substituição de átomos do tipo " $A$ ", que compõem o sistema de multicamadas, por átomos do tipo " $B$ ", chamados de impurezas, onde os átomos " $A$ " e " $B$ " são metais de transição.

O artigo está organizado da seguinte forma: na seção 2 descrevemos o hamiltoniano do sistema e as simplificações adotadas na sua modelagem; definimos na seção 3 a densidade de estados em termos da função de Green e apresentamos o método de dizimação; a seção 4 é destinada ao tratamento do sistema com impureza; na seção 5 , apresentamos o cálculo da energia de segregação, os resultados e as considerações finais.

\section{Modelagem do Sistema}

Os metais de transição são representados pelas séries $3 d, 4 d$ e $5 d$ que se encontram no centro da tabela periódica [5]. A estrutura eletrônica desses metais pode ser analisada com base na estrutura eletrônica atômica da seguinte forma:

$$
\text { [estados mais internos] }+n d^{m}(n+1) s^{r},
$$

onde $n=3,4,5, r=1,2$ e $m=1, \ldots, 10$ ao longo de cada uma das séries $3 d, 4 d$ e $5 d$.

Podemos supor que os estados mais internos não se alteram significativamente quando se passa do átomo para o sólido, e os estados $\mathbf{s}$ da última camada, por serem fracamente ligados aos átomos, dão origem a um gás de elétrons quase livres. Por outro lado, os orbitais d dão origem a estados com um razoável grau de localização nos átomos de origem.

Para descrevermos os estados eletrônicos, formados a partir dos orbitais d, podemos empregar o método de combinação linear dos orbitais atômicos (LCAO) [3]. Esse é um método parametrizado e consiste em descrever os estados eletrônicos de um sólido a partir das funções e energias atômicas. A ideia deste método é escrever uma função de onda para o sistema como uma combinação linear dos orbitais atômicos centrados em cada sítio.

O hamiltoniano $[7,8]$ que utilizamos para descrever o sistema tem a seguinte 
forma:

$$
H_{A}=\sum_{\vec{R}_{i}, \mu}\left|\vec{R}_{i}, \mu>\epsilon_{i}^{\mu}<\vec{R}_{i}, \mu\right|+\sum_{\vec{R}_{i}, \vec{R}_{j}} \sum_{\mu, \nu}\left|\mu, \vec{R}_{i}>t_{i, j}^{\mu, \nu}<\nu, \vec{R}_{j}\right|,
$$

onde $\epsilon_{i}^{\mu}$ representa a energia do orbital atômico $\mu$, no sítio $\vec{R}_{i}$ e $t_{i, j}^{\mu, \nu}$ a integral de transferência, que representa a probabilidade de um elétron "saltar" do sítio $i$ no orbital $\mu$ para o sítio $j$ no orbital $\nu$. Na notação de Dirac, o vetor de estado $\mid i>$ representa uma matriz linha do espaço de Hilbert, enquanto que $<i \mid$ representa um elemento (matriz coluna) do espaço dual.

Nesse trabalho, nossa atenção estará dirigida apenas sobre os orbitais d; desprezamos os efeitos da hibridização destes com o orbital s-p. Por simplicidade, consideramos nesse modelo apenas um orbital por sítio.

Os sistemas que estudamos apresentam simetria de translação ao longo de direções paralelas aos seus planos cristalinos, e que esquematicamente podem ser representados pela Figura 1.

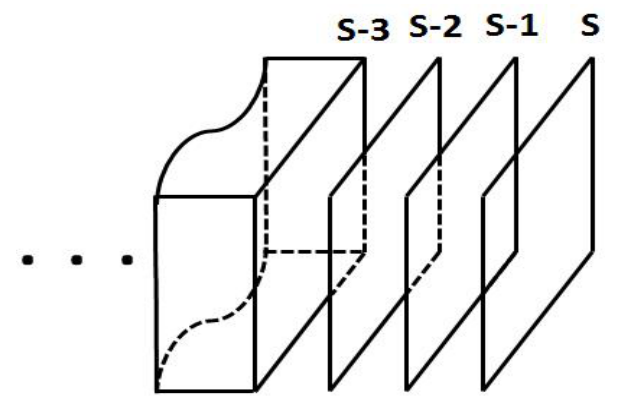

Figura 1: Representação esquemática do sistema de multicamadas metálicas.

Para descrevermos a estrutura desse tipo de sistema, tomamos a transformada de Fourier bidimensional dos elementos de matriz do hamiltoniano, com

$$
\left|\vec{k}, l, \mu>=\frac{1}{\sqrt{N_{\|}}} \sum_{j} e^{-i \vec{k} \cdot \vec{R}_{j}}\right| \vec{R}_{j}, \mu>
$$

Assim, passamos para uma representação mista, caracterizada pelo vetor de onda $\vec{k}$, paralelo aos planos, e pelo índice de plano $l$. O vetor $\vec{k}$ está restrito à primeira zona de Brillouin bidimensional, correspondente à estrutura cristalina (também bidimensional) definida pelas posições atômicas dos planos cristalinos.

$\mathrm{Na}$ representação mista, o hamiltoniano pode ser escrito na forma

$$
H_{A}=\sum_{\vec{k}}\left\{\sum_{l} \epsilon_{l}(\vec{k})|l \vec{k}><l \vec{k}|+\sum_{l \neq l^{\prime}} t_{l l^{\prime}}(\vec{k})\left|l \vec{k}><l^{\prime} \vec{k}\right|\right\}
$$

onde

$$
\epsilon_{l}(\vec{k})=\epsilon_{0}+\sum_{j} t\left(\overrightarrow{R_{\| j}}, 0\right) e^{-i \vec{k} \cdot \overrightarrow{R_{j}}} \quad \text { e } \quad t_{l l^{\prime}}(\vec{k})=\sum_{j} t\left(\overrightarrow{R_{\| j}}, l-l^{\prime}\right) e^{-i \vec{k} \cdot \overrightarrow{R_{j}}}
$$


com $\vec{R}_{\| j}$ especificando a posição de cada átomo em um plano $l[1]$.

Dessa forma, para cada $\vec{k}=\left(k_{x}, k_{y}, 0\right)$ paralelo, temos um hamiltoniano correspondente a um sistema unidimensional com energias atômicas $\epsilon_{l}(\vec{k})$ e integrais de transferência $t_{l l^{\prime}}(\vec{k})$.

\section{Funções de Green e Densidade de Estados}

A descrição eletrônica de um sistema semi-infinito pode ser feita através das funções de Green [12], definidas em termos do hamiltoniano como

$$
G(\omega)=[\omega-H]^{-1},
$$

onde os seus elementos de matriz tem a forma

$$
G_{l l}(\omega, \vec{k})=[\omega-H(\vec{k})]_{l l}^{-1} .
$$

A densidade de estados do sistema [1] é definida em termos das funções de Green através da expressão

$$
\rho(\omega)=-\frac{1}{\pi} \operatorname{Im} \sum_{l, \vec{k}} G_{l l}(\omega, \vec{k})=\sum_{l} \rho_{l}(\omega),
$$

onde o $\rho_{l}(\omega)$ é a densidade local de estados no sítio $l[2]$.

Como vimos, com a representação mista, o hamiltoniano torna-se diagonal em $\vec{k}$. Dessa forma, obtemos uma soma em $\vec{k}$, onde para cada $\vec{k}$, temos um hamiltoniano correspondente a um sistema unidimensional. Estes sistemas unidimensionais podem ser tratados com base no método de dizimação [10]. Esse método consiste na redução progressiva dos graus de liberdade do sistema, de tal forma que os parâmetros do hamiltoniano são renormalizados a cada iteração. O processo de renormalização, após um certo número de iterações, nos leva a um ponto fixo, caracterizado por uma energia $\epsilon^{*}$ e uma integral de transferência $t^{*}$ [14].

Considerando, por exemplo, um sistema infinito unidimensional, (Figura 2) as equações de renormalização para os parâmetros do hamiltoniano, na iteração (renormalização) " $j$ ", tem a forma

$$
\epsilon^{j}=\epsilon^{(j-1)}+2 \frac{\left[t^{(j-1)}\right]^{2}}{\omega-\epsilon^{(j-1)}} \quad \text { e } \quad t^{j}=\frac{\left[t^{(j-1)}\right]^{2}}{\omega-\epsilon^{(j-1)}} .
$$

A Figura 3 representa a rede cúbica simples, considerando as direções (100) e (110), e as Figuras 4 e 5 representam, respectivamente, as densidades de estados locais a partir da superfície em direção ao volume.

Os autovalores do hamiltoniano fornecem a estrutura de banda e, para a rede cúbica simples, com os vizinhos mais próximos localizados em $( \pm a, 0,0),(0, \pm a, 0)$ e $(0,0, \pm a)$, tem a forma geral

$$
\epsilon(\vec{k})=2 t\left(\cos \left(k_{x} a\right)+\cos \left(k_{y} a\right)+\cos \left(k_{z} a\right)\right),
$$




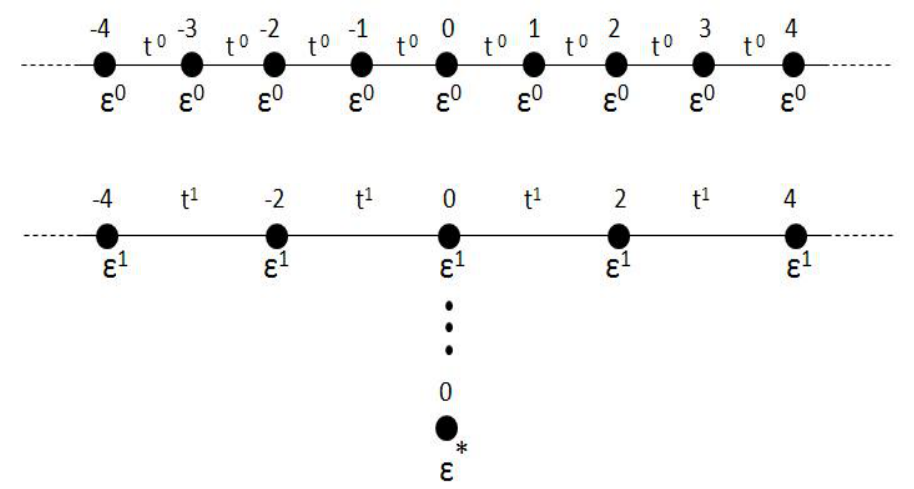

Figura 2: Representação esquemática do processo de dizimação.
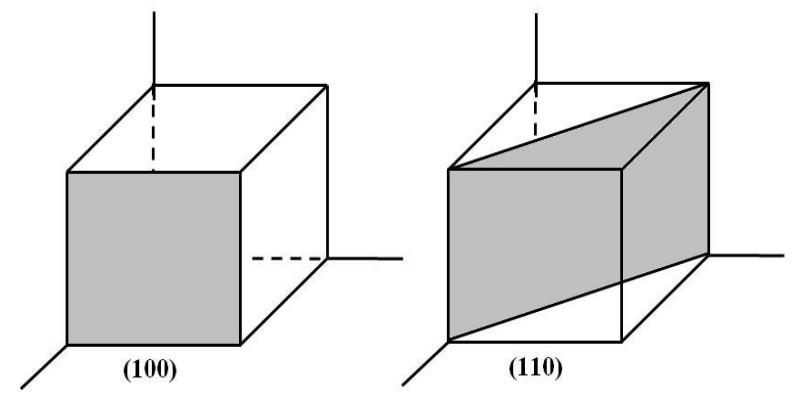

Figura 3: Representação esquemática da rede cúbica simples, mostrando as direções (100) e (110).

onde $a$ é parâmetro de rede [7]. Nos cálculos, consideramos $a=1$ e $t=1$.

Na Figura 4, notamos que para as superfícies, tanto na direção (100) como na direção (110), os estados eletrônicos estão concentrados na região central da banda, quando comparados com o volume, o que decorre da redução do número de átomos vizinhos. Ainda em relação às superfícies, observamos também o desaparecimento das singularidades de Van Hove ( $\vec{k}$ na zona de Brillouin tal que $\left.\nabla_{\vec{k}} \epsilon(\vec{k})=0\right)[3]$ associadas à perda de simetria de translação na direção perpendicular aos planos [8].

Verificamos também que a medida que consideramos camadas mais internas, nas duas direções, as curvas de densidades de estados locais tendem para a do volume e que estas curvas exibem oscilações. Observa-se claramente que as amplitudes e comprimentos de onda associados a essas oscilações diminuem à medida em que nos afastamos da superfície.

Podemos calcular então a estrutura eletrônica de sistemas de multicamadas metálicas e como esta é afetada pela introdução de impurezas substitucionais em planos a partir da superfície. 

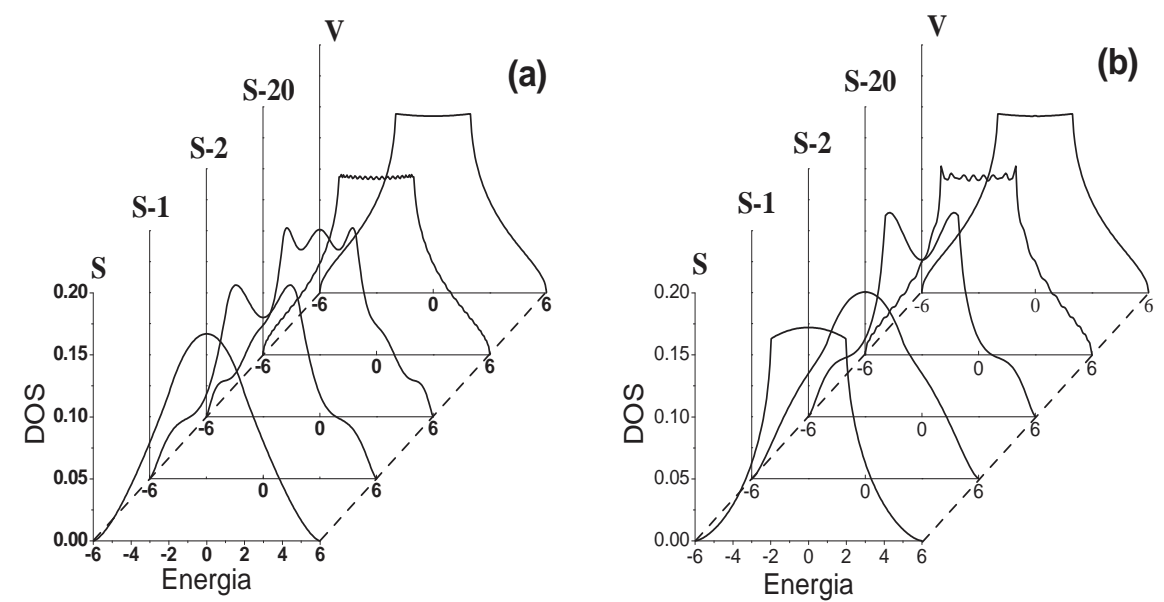

Figura 4: Densidades de estados para a rede cúbica simples nas direções (a) (100) e (b) (110) para a superfície (S), planos subsuperficiais (S-1), (S-2), (S-20) e volume (V).

\section{Sistemas com Impurezas}

Investigamos o efeito de uma única impureza $B$ em um sistema de camadas constituído por átomos do tipo $A$, ou seja, ligas da forma $A_{1-x} B_{x}$, para $x<<1$ (limite diluído). A impureza é colocada em um sítio pertencente a um determinado plano paralelo à superfície. Nesse caso, o hamiltoniano passa a ser escrito como $H=$ $H_{A}+V$, onde $H_{A}$ é o hamiltoniano do cristal puro e $V=|i>\Delta \epsilon<i|$, sendo $\Delta \epsilon \mathrm{o}$ potencial espalhador associado à impureza no sítio $i$ [8].

Consideramos também que a variação no potencial eletrônico $\Delta \epsilon$, devido à introdução da impureza, é restrita ao sítio em que ela está localizada. A função de Green associada à $H$ satisfaz a equação de Dyson $G=G^{A}+G^{A} V G$, onde $G^{A}$ é a função de Green do cristal puro. O potencial $\Delta \epsilon$ pode ser calculado através da regra de soma de Friedel [9], a qual fornece uma relação entre esse potencial e a diferença de valência $\Delta n=n^{B}-n^{A}$ entre os átomos $A$ e $B$, ou seja,

$$
\Delta n=\int_{-\infty}^{E_{F}} d \omega f_{F D}(\omega, T) \Delta \rho_{l}(\omega)=-\frac{1}{\pi} \operatorname{Im} \int_{-\infty}^{E_{F}} d \omega \frac{1}{1+e^{\frac{\omega-E_{F}}{k T}}} \Delta G_{l l}(\omega),
$$

onde $f_{F D}(\omega, T)$ é a função distribuição de Fermi-Dirac, $\Delta \rho(\omega)=\rho_{l}(\omega)-\rho_{l}^{A}(\omega)$ e $\Delta G(\omega)=G(\omega)-G^{A}(\omega)$,

$$
f_{F D}=\frac{1}{1+e^{\frac{\omega-E_{F}}{k T}}} \quad \text { e } \quad \Delta G_{l l}(\omega)=-\frac{\Delta \epsilon_{l}}{1-\Delta \epsilon_{l} G_{l l}^{A}(\omega)},
$$

com $\rho_{l}(\omega)$ e $G_{l l}(\omega)$ representando, respectivamente, a densidade de estados e a função de Green do sistema com impureza. Temos então que

$$
\Delta n=\frac{1}{\pi} \operatorname{Im} \int_{-\infty}^{E_{F}} d \omega \frac{1}{1+e^{\frac{\omega-E_{F}}{k T}}} \frac{\Delta \epsilon}{1-\Delta \epsilon G_{l l}^{A}(\omega)} \frac{d G_{l l}^{A}(\omega)}{d \omega},
$$


donde

$$
\Delta n=-\frac{1}{k T \pi} \operatorname{Im} \int_{-\infty}^{E_{F}} d \omega \ln \left|1-\Delta \epsilon_{l} G_{l l}^{A}(\omega)\right| \frac{e^{\frac{\omega-E_{F}}{k T}}}{\left[1+e^{\frac{\omega-E_{F}}{k T}}\right]^{2}} .
$$

Com a determinação do potencial espalhador, podemos determinar a variação na energia de segregação, definida aqui como a variação na energia total $\Delta E_{l}$ de um sistema semi-infinito devido à introdução de uma impureza substitucional, na superfície e em planos subsuperficiais [4, 1], cuja expressão é dada por

$$
\Delta E_{l}=E_{l}-E_{l}^{A}=\int_{-\infty}^{E_{F}} d \omega f_{F D}(\omega, T) \omega \Delta \rho_{l}
$$

Fazendo as substituições já realizadas no cálculo do potencial em termos da diferença de valência, obtemos

$$
\Delta E_{l}=\frac{1}{\pi} \operatorname{Im} \int_{-\infty}^{E_{F}} d \omega \omega \frac{1}{1+e^{\frac{\omega-E_{F}}{k T}}} \frac{\Delta \epsilon}{1-\Delta \epsilon G_{l l}^{A}(\omega)}\left[G_{l l}^{A}(\omega)\right]^{2} .
$$

Estamos interessados em comparar a variação de energia quando a impureza se encontra em um plano $l$, com aquela correspondente à impureza localizada no "volume". Assim, devemos calcular $\delta E_{l}=\Delta E_{l}-\Delta E_{\text {vol }}$.

A presença da função de Fermi-Dirac nas expressões (4.3) e (4.6) é responsável pelo surgimento de pólos. Dessa forma, fazemos uma integração usando o método dos resíduos. No entanto, para obtermos um melhor entendimento do fenômeno de interferências quânticas que ocorrem no sistema, e seus efeitos na segregação, fazemos uma aproximação, como veremos na próxima seção, tomando o limite em que a temperatura tende a zero.

\section{Cálculo da Energia de Segregação e Resultados}

Como mencionamos na seção anterior, consideramos $T=0$, o que corresponde a $f_{F D}(\omega, t)=1$. Assim, de (4.3), obtemos

$$
\Delta n=\frac{1}{\pi} \operatorname{Im} \int_{-\infty}^{E_{F}} d \omega \frac{\Delta \epsilon}{1-\Delta \epsilon G_{l l}^{A}(\omega)} \frac{d G_{l l}^{A}(\omega)}{d \omega}=-\frac{1}{\pi} \operatorname{Im}\left\{\ln \left[1-\Delta \epsilon_{l} G_{l l}^{A}\left(E_{F}\right)\right]\right\}
$$

$\mathrm{Ou}$

$$
\Delta \epsilon_{l}=\frac{\operatorname{tg}(\pi \Delta n)}{\operatorname{Re}\left(G_{l l}^{A}\left(E_{F}\right)\right) \cdot \operatorname{tg}(\pi \Delta n)+\operatorname{Im}\left(G_{l l}^{A}\left(E_{F}\right)\right)} .
$$

A Figura 5 mostra os perfis dos potenciais associados à impureza nos diversos planos a partir da superfície em direção ao volume. Consideramos as direções de crescimento (100) e (110), com a ocupação eletrônica $n_{A}=0.94$ para a rede hospedeira, e impurezas com ocupações de $n_{B}=0.54$ e $n_{B}=0.84$. Esses valores correspondem, no modelo simplificado adotado, aos elementos $\mathrm{Ni}, \mathrm{Cr}$ e $\mathrm{Co}$, respectivamente, na série $3 d$ da tabela periódica. 

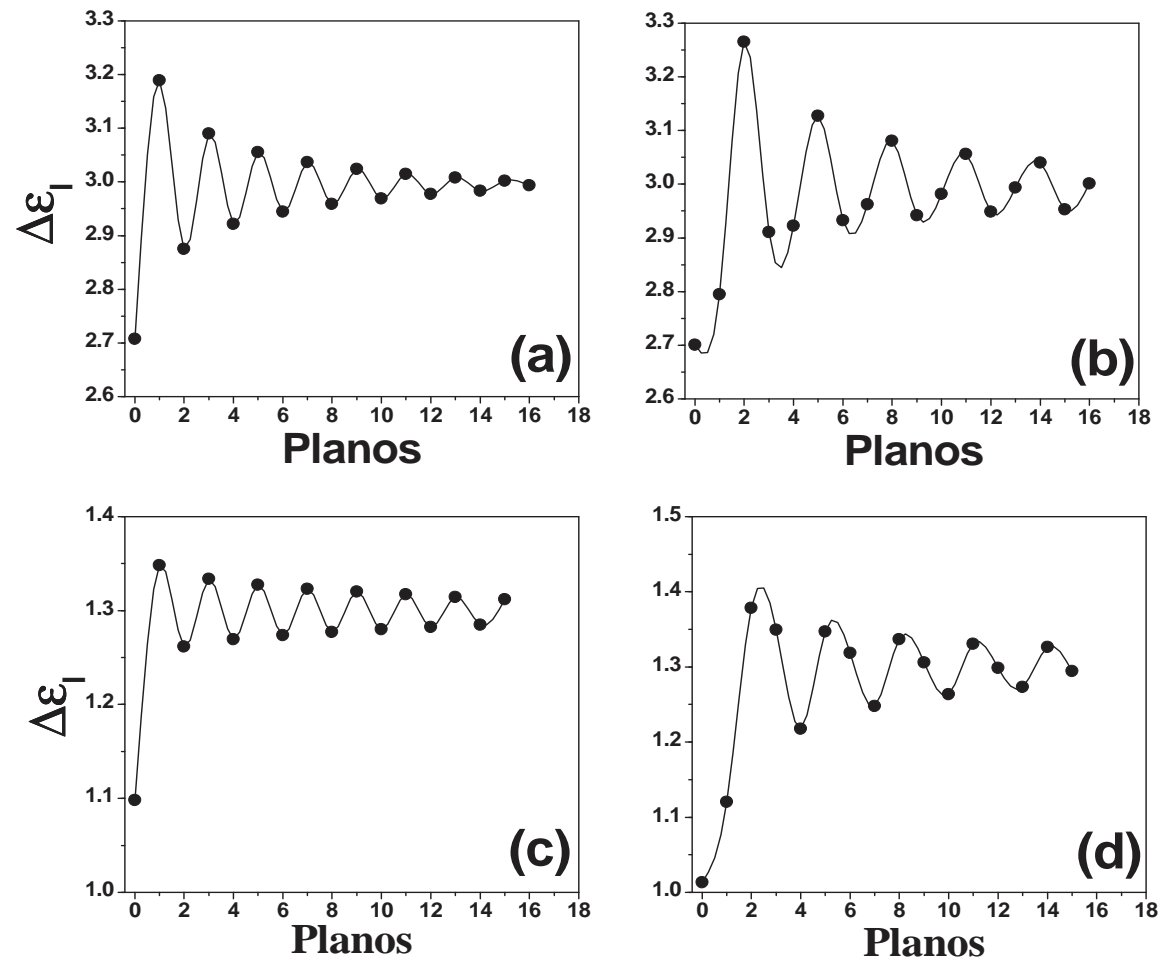

Figura 5: Potenciais associados à impureza para a superfície, indicada pelo índice 0 nos gráficos, e planos subsuperficiais, para a rede cúbica simples nas direções (a) (100) e (b) (110), considerando $n_{A}=0.94$ e $n_{B}=0.54$ e nas direções (c) (100) e (d) (110), considerando $n_{A}=0.94$ e $n_{B}=0.84$. As linhas são guias para os olhos.

Nesse exemplo apresentado, as ocupações dos elementos considerados foram obtidas a partir do fato que na teoria de elétrons itinerantes, suas bandas $d$ possuem 9.4 elétrons para o $\mathrm{Ni}, 8.4$ para o $\mathrm{Co}$ e 5.4 para o $\mathrm{Cr}$. No entanto, estamos considerando os orbitais $d$ como degenerados (degenerescência quíntupla) e independentes e, além disso, não levamos em conta a polarização de spin, o que nos permite escrever a ocupação de um orbital por sítio, para cada um desses elementos, como a décima parte da ocupação real. Vale mencionar que essa modelagem nos permite apenas fazer uma comparação qualitativa com dados experimentais.

Observamos que, em relação ao volume, tanto para o $C r$, como para o $C o$, os valores de $\Delta \epsilon_{l}$ variam quando são considerados planos subsuperficiais e, de forma mais acentuada, na superfície. Notamos também que a amplitude da oscilação diminui na medida em que a impureza se afasta da superfície.

A oscilação observada no potencial espalhador de plano para plano, à medida em que tomamos planos mais internos, decorre das interferências quânticas entre as ondas eletrônicas incidentes com as refletidas pela superfície. Uma consequên- 
cia desse fenômeno de interferência é que a amplitude dos estados eletrônicos fica modulada próxima à superfície. Essa oscilação na amplitude fica evidente através da oscilação da densidade de estados local, em torno do valor do volume, à medida que são consideradas camadas mais internas, como vimos na seção 3 .

A variação na densidade de estados local também se verifica na energia de Fermi, o que traz uma importante consequência para o problema da impureza, pois a blindagem do potencial devida à impureza depende da densidade de estados local no nível de Fermi. Dessa forma, a energia eletrônica do sistema, que também depende da densidade de estados no nível de Fermi, deve oscilar quando a impureza se desloca da superfície em direção ao volume.

Consideramos agora a variação de energia total $\Delta E_{l}$, de um sistema semi-infinito, devido à introdução de uma impureza substitucional, na superfície e em planos subsuperficiais [4, 1], cuja expressão é dada por

$$
\Delta E_{l}=E_{l}-E_{l}^{A}=\int_{-\infty}^{E_{F}} d \omega f_{F D}(\omega, t) \omega \Delta \rho_{l} .
$$

Estamos interessados em comparar a variação de energia quando a impureza se encontra em um plano $l$, com aquela correspondente à impureza localizada no volume. Portanto, calculamos

$$
\delta E_{l}=\Delta E_{l}-\Delta E_{v o l}=\int_{-\infty}^{E_{F}} d \omega f_{F D}(\omega, t) \omega\left[\Delta \rho_{l}-\Delta \rho_{v o l}\right] .
$$

Consideramos $T=0$, o que corresponde a $f_{F D}(\omega, t)=1$, e $n_{A}=0,94 \operatorname{com} n_{B}=$ 0,54 e $n_{B}=0,84$. Procuramos, assim, determinar quais os planos energeticamente mais favoráveis para se encontrar a impureza, o que deve corresponder a um mínimo local de energia. Esperamos que, da mesma forma que ocorre com a variação do potencial, mostrada na Figura 5, a variação de energia eletrônica total seja também função da posição da impureza.

Verificamos que a variação da energia eletrônica total do sistema oscila em função da posição da impureza e que esse comportamento oscilatório é coerente com os perfis de oscilação verificados nas curvas dos potenciais. A origem dessa oscilação é a presença da superfície e, como já mencionamos, o fenômeno de oscilação quântica provocado por ela. Esse comportamento é verificado nas duas direções consideradas. No entanto, a energia de segregação oscila de forma diferente quando a posição da impureza se afasta da superfície, exibindo ambas uma diminuição na amplitude de oscilação.

Pode-se notar nos gráficos (b) e (d) da Figura 6, relacionados à direção (110), uma curva com período de oscilação complexo, o que guarda relação com a forma com que ocorre a blindadem das impurezas substitucionais nos diversos planos nessa direção, que por sua vez está relacionada com a variação da densidade de estados no nível de Fermi. Nos gráficos (a) e (c) relacionados à a direção (100), notamos que a superfície é o local mais favorável energeticamente à localização da impureza e que o menos favorável é o primeiro plano subsuperficial. Quando tomamos a direção (110), verificamos que a superfície ainda é o local mais favorável à localização da impureza e o segundo plano subsuperficial é o menos favorável. 

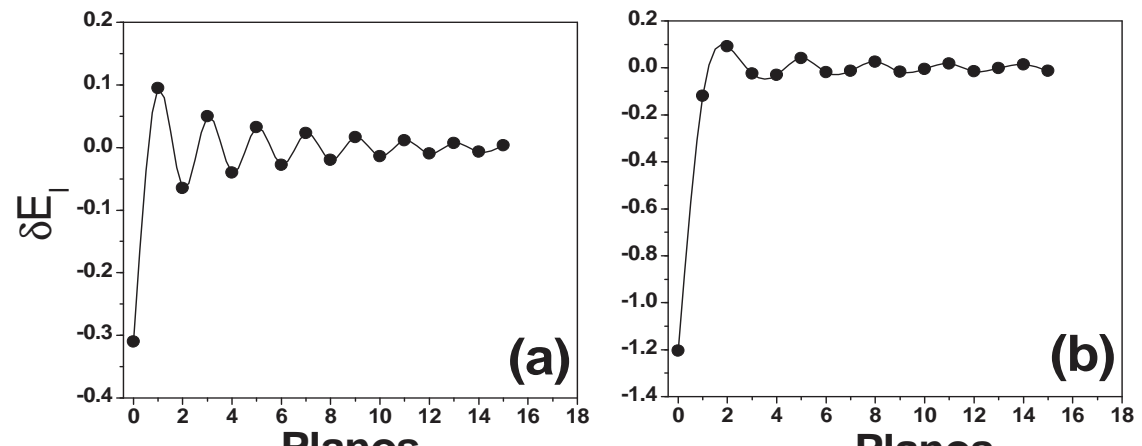

Planos
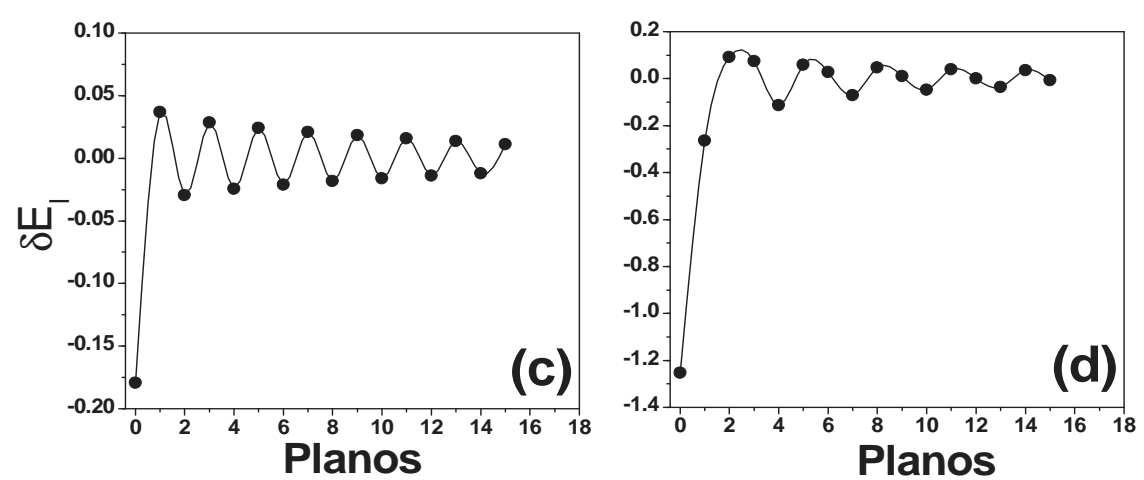

Figura 6: Variação da energia $\delta E_{l}$ associada à impureza para a superfície, indicada pelo índice 0 nos gráficos, e planos subsuperficiais, para a rede cúbica simples nas direções (a) (100) e (b) (110), considerando $n_{A}=0.94$ e $n_{B}=0.54$ e nas direções (c) (100) e (d) (110), considerando $n_{A}=0.94$ e $n_{B}=0.84$. As linhas são guias para os olhos.

Investigamos a contribuição da variação na energia eletrônica ao complexo problema de segregação de uma liga metálica diluída. Nesse estudo, ficou estabelecida a importância da presença da superfície e das interferências quânticas decorrentes das ondas eletrônicas incidentes com as refletidas por ela. Esses resultados indicam a possibilidade de ocorrer modulação na concentração de ligas, na região próxima à superfície.

\section{Referências}

[1] J. d'Albuquerque e Castro, A.C. de Castro Barbosa, M.V. Tovar Costa, Quantum interference effects on the segregation energy in diluted metallic alloys, Phys. Rev. B, 70, No. 16 (2004), 165415.

[2] G. Allan, P. Lenglart, Electronic Surface States in Cubic Lattice, Surf. Sci., 30 (1972), 641-652. 
[3] N.W. Ashcroft, N.D. Mermin, "Física do Estado Sólido", Cengage Learning, São Paulo, 2011.

[4] A.C. de Castro Barbosa, J. d'Albuquerque e Castro, Eletronic structure of substitutional impurities near metallic surfaces, J. Magn. Magn. Mat., 121 (1993), 167-169.

[5] A.L. Companion, "Ligação Química", Edgard Blücher, São Paulo, 1970.

[6] A.T. Costa Jr., A.C. de Castro Barbosa , J. d'Albuquerque e Castro, R.B. Muniz, Effects of impurities on the exchange coupling in magnetic metallic multilayers, J. Phys.: Condens. Matter, 13 (2001), 1827-1837.

[7] E, N, Economou, "Green's Function in Quantum Physics", Springer Series in Solid State Science, Springer-Verlag, vol. 7, Berlin, 2010.

[8] H. Ehrenreich, D. Turnbull, "Solid State Physics: Advances in Research and Applications", vol. 23, Academic Press, New York, 1969.

[9] J. Friedel, Metallic alloys, Nuovo Cimento, 7 (1958), 287-311.

[10] C.E.T. Gonçalves da Silva, B. Koiller, Local density of states in a disordered chain: a renormalization group approach, Solid State Communications, 40 (1981), 215-219.

[11] D.G. Teixeira, A.C. de Castro Barbosa, M.V. Tovar Costa, Modelagem de segregação de impurezas em ligas metálicas, em Congresso de Matemática Aplicada e Computacional, Uberlândia, MG, (2011).

[12] D. Kalkstein, P. Solven, A Green's function theory of surface science, Surf. Sci., 26 (1971), 85-89.

[13] J. Kudrnovsky, V. Drchal, I. Turek, P. Weinberger, Phys. Rev B, 50 (1994), 16105-16108.

[14] M.P. Lopez Sancho, J. M. Lopez Sancho e J. Rubio, Highly convergent schemes for the calculation of bulk and surface Green functions, J. Phys. F: Met. Phys., 15 (1985), 851-858.

[15] L.E. Orgel, "Introdução a Química dos Metais de Transição", Edgard Blucher, (1970).

[16] L.M. Rozanov, M.H. Hablanian, "Vacuum Technique", Taylor \& Francis, London; New York, 2002.

[17] T. Shinjo, T. Takada, "Studies in Physical and Theoretical Chemistry: Metallic Superlattices", Elsevier Science, Amsterdam, 1987.

[18] J. Slater, G.F. Koster, Simplified LCAO method for the periodic problem, Phys. Rev., 94 (1954), 1498-1524. 
[19] R.V. Stuart, "Vacuum Technology: thin Films and Sputtering - an introduction", Academic Press, 1987.

[20] T.T. Tsong, M. Ahmad, Oscillatory surface segregation of $\mathrm{Pt}-\mathrm{Rh}(\mathrm{S})$ alloys, Phys. Rev. B, 42 (1990), 1464-1466.

[21] T.T. Tsong, Time-of-flight atom-probe field ion microscope studies of surfacerelated phenomena, Surface and Interface Analysis, 36 (2004), 365-371. 